\title{
Evaluation of Bleach Sedimentation Technique in Detection of Mycobacterium tuberculosis by LED Microscopy
}

\author{
Suneet K. Yadav ${ }^{1}$, R.Sujatha ${ }^{2 *}$, D.N. Singh ${ }^{3}$ and Deepak Sameer ${ }^{4}$ \\ Department of Microbiology, Rama Medical College Hospital and Research Centre, \\ Mandana, Kanpur, India \\ *Corresponding author
}

\begin{tabular}{|l|}
\hline Ke y w o r d s \\
Tuberculosis, Acid \\
fast bacilli, Ziehl- \\
Neelsen method, \\
Aura-mine method, \\
Light emitting \\
diode, Sodium \\
hypochlorite
\end{tabular}

The bacteriological diagnosis of tuberculosis (TB) is largely dependent on the Ziehl-Neelsen (ZN) microscopy. This method has a low sensitivity, which can be improved by the concentration of the specimen with sodium hypochlorite or Bleach $(\mathrm{NaOCl})$, followed by sedimentation after doing LED. This study was conducted to evaluate the efficiency of bleach sedimentation technique in detection of Mycobacterium tuberculosis by LED microscopy. A Total of 100 sputum samples from the patients who visited RNTCP of Rama Medical College Hospital and Research Centre between April 2015 and March 2016. Morning samples were collected from each patient, direct smears were prepared and they were stained with the ZN Stain and aura LED technique and the remaining samples were concentrated by using $3.5 \%$ $\mathrm{NaOCl}$, followed by sedimentation and staining with $\mathrm{ZN}$ stain and aura LED stain. The improvement in the sensitivity following the bleach method was studied. Out of 100 sputum samples, 50 smear positive samples by $\mathrm{ZN}$ showed $100 \%$ positivity but increase the grading of positive result after the bleach sedimentation technique in both LED and ZN microscopy while 50 AFB negative samples shows $12 \%$ positivity rate was $\mathrm{ZN}$ for bleach and $26 \%$ shows positivity rate was using aura LED for bleach sedimentation technique. Whereas the overall positivity rate was $56 \%$ shows by $\mathrm{ZN}$ for bleach and $63 \%$ shows positivity rate was using aura LED for bleach sedimentation technique. A statistically significant $(p<0.0001)$ increase in the positivity with the use of the bleach method was detected as compared to that with the use of the direct ZN staining and aura LED Method. After bleach for LED is sensitivity, specificity, positive predictive value (PPV), and the negative predictive value (NPV) were $100 \%, 89.3 \%$, $90.3 \%$ and $100 \%$ respectively with a $95 \%$ confidence interval, with the use of the $3.5 \% \mathrm{NaOCl}$ method. In the present study, the Bleach sedimentation method increases the positivity in aura LED microscopy when it was compared with the $\mathrm{ZN}$ microscopy The bleach method has advantages over the direct method, as it is simple and as it does not require any additional expertise beyond that which is required for the conventional direct smear microscopy. The materials and the reagents are also affordable and they are available locally.

\section{Introduction}

Tuberculosis is one of the most prevalent infection of human beings and contributes considerably to illness and death around the world. It is estimated by WHO that $1 / 3^{\text {rd }}$ of the global population is infected with $\mathrm{TB}^{1}$ and it is the second leading cause of death from an infection worldwide after HIV. Annually approximately 3 million death by TB, about 5 
death every minute and 8-10 million people are affected with Mycobacterium tuberculosis. ${ }^{2}$ In India, tuberculosis (TB) accounts for one-fifth of the global TB incident cases. Each year nearly 2 million people develop TB in India, of which around 0.87 million are infectious cases and annually around 3, 30,000 Indians die due to TB. ${ }^{3}$

According to Global TB Report 2013, the new data confirm that the world is on track to meet the 2015 UN Millennium Development Goals (MDGs) target of reversing TB incidence, along with the target of a $50 \%$ reduction in the mortality rate by 2015 (compared to 1990). ${ }^{4}$ Although, WHO estimated that if better precautions are not to be taken to tackle this disease, nearly 1 billion people will be infected between 2000 and 2020. ${ }^{5}$

Therefore, the early diagnosis of tuberculosis is crucial first step for tuberculosis control program worldwide especially in the wake of emergence of drug resistant TB and its related implications for HIV infected patients. ${ }^{6}$ This goal is achieved by Serial sputum smear microscopy to confirm TB. Unfortunately, TB microscopy is associated with low and variable sensitivity, particularly in high HIVprevalence settings. A number of research groups have aimed to improve the performance of smear microscopy through new technology and service delivery approaches. ${ }^{7}$

A microbiological approach in the diagnosis of pulmonary TB is comparatively new than the radiology but still hold equal significance, microbiologist are giving newer better way to diagnose as well as eradicate the disease, In includes a wide range of procedure which ensure rapidly as well as accuracy in the detection and isolation of Myco-TB.

The acid fast smear has been used as an aid in the diagnosis of mycobacterial disease of many years. It is the simplest procedure currently available to detect AFB in clinical sample by $\mathrm{ZN}$ - Staining. ${ }^{8} \mathrm{~A}$ part for $\mathrm{ZN}-$ Staining is being advised by RNTCP as provided to the state designated Intermediate Reference Laboratories (IRLS) under Revised National Tuberculosis Control Programmed (RNTCP).

The WHO Strategic and Technical Advisory Group (STAG) for TB recommended that fluorescence microscopy (FM) using lightemitting diode (LED) be phased in as an alternative for conventional Ziehl-Neelsen (ZN) microscopy. In addition to $10 \%$ improvement of sensitivity when using conventional FM compared to $\mathrm{ZN}$ microscopy with comparable specificity, recent advances of simple LED-based FM systems can allow implementation of FM in low levels of health service ${ }^{9}$.

There are various concentration method for improving sensitivity of direct microscopy for detection of tubercle bacilli in specimen. ${ }^{10}$ Bleach sedimentation method of detection of tubercle bacilli has been recently described for sputum specimens and studies have shown improved detection. ${ }^{11}$ Sputum processing methods using bleach sedimentation (sodium hypochlorite $[\mathrm{NaOCl}$ before smear microscopy were also identified as promising approaches to improve performance of smear microscopy.

Among these approaches, overnight $\mathrm{NaOCl}$ sedimentation has been considered suitable for lower levels of health services. In a recent systematic review (2010) of studies evaluating the accuracy of different processing methods compared to direct $\mathrm{ZN}$ microscopy, and using TB culture as reference standard, bleach sedimentation was $9 \%$ more sensitive than direct microscopy. A study in Kenya has also reported a 23\% increase in case-detection yield. ${ }^{7}$ 


\section{Materials and Methods}

A cross sectional study was conducted in the Department Of Microbiology of Rama Medical College Kanpur to compare the effect of bleach on sputum followed by sedimentation (bleach concentration method) and direct sputum smear microscopy (direct method) with LED and ZN microscopy, between April 2015 and March 2016.

Samples was collected in sterile Widemouthed, screw capped translucent container (at least $35 \mathrm{~mm}$ in diameter) so that the patient can expectorate easily inside the container without contaminating the outside and to observe specimen volume and quality without opening the container. Recently Sputum specimen ideally, having a volume of $4-5 \mathrm{ml}$, although smaller quantities was acceptable if the quality is satisfactory (indicated by mucoid or mucopurulent material).It is best to obtain sputum early in the morning before the patient has eaten or taken medication. ${ }^{12}$

Specimens will be transported to the laboratory as soon as possible after collection. If delay is unavoidable, the specimens was refrigerated at $4{ }^{\circ} \mathrm{C}$ to inhibit the growth of unwanted microorganisms 24 hours. ${ }^{12}$

A new unscratched slide will be selected and was labeled with the laboratory serial number with a diamond marking pencil. Two smear on two slides was made from sediment by the sterilized inoculating loop. A good smear is spread evenly, $2 \mathrm{~cm} \times 3 \mathrm{~cm}$ in size and is neither too thick nor too thin. The slide was allowed to air dry for 15-30 minutes. The slide will be fixed by passing it over a flame 3-5 times for 3-4 seconds each time ${ }^{12}$ and one smear stained for using the (Direct method) standard Ziehl- Neelsen (ZN) technique and second smear stained with LED technique. The stained slides were examined for AFB by expertise medical laboratory technologist.
The remaining portions of the $4-5 \mathrm{ml}$ sputum will be transferred to a $15-\mathrm{mL}$ disposable plastic conical tube with an equal volume of neat 3.5\% commercial bleach (prepared in distilled water) and will be left on the bench for overnight sedimentation.

After sedimentation, the supernatant will be poured off and one to two drops of sediment will be transferred to a slide using a sterile glass pipette. Two smear will be made and stained using the LED and ZN staining. ${ }^{8}$ The result was reported according to WHO guidelines and graded negative, scanty, $1+, 2+$ or $3+$ depending on the amount of bacilli. ${ }^{12}$

The data was analyzed by using a statistical

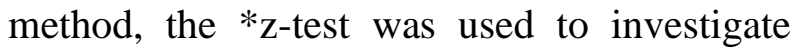
differences in proportions. A $P$ - value less than $0.0001^{*}$ was considered as statistical significant. The project received Ethical clearance from the institutional Ethical clearance RMCH\&RC, Mandhana Kanpur.

\section{Results and Discussion}

Out of total 100 samples, 50 cases were smear positive and 50 cases were smear negative by Direct ZN Staining method. From selected 100 sputum samples, 57\% showed the smear positivity after Bleach Concentration. $100 \%$ positivity was observed in smear positive sample while $14 \%(n=7)$ positivity reveals in smear negative sample (Table 1).

All the positive samples by the direct method were positive by the bleach sedimentation method but, $56 \%(\mathrm{n}=6)$ showed positive in case of $\mathrm{ZN}$ after bleach instead of $63 \%(\mathrm{n}=13)$ showed positivity in LED after bleach (Table $3)$.

Table 4 demonstrates the effect of relative direct and bleach method (ZN / LED) on smear grading from the sputum sample. In $\mathrm{ZN}$ staining for direct- $12 \%$ were with scanty, 
$36 \%$ were with $1+, 38 \%$ were with $2+, 14 \%$ were with $3+$ grades, while $\mathrm{ZN}$ after bleachincreased the grading i.e. $10 \%$ were with scanty, $26 \%$ were with $1+, 44 \%$ were with $2+$, $20 \%$ were with $3+$ grades. in LED for direct$10 \%$ were with scanty, $34 \%$ were with $1+$, $42 \%$ were with $2+, 14 \%$ were with $3+$ grades, while LED for bleach showed $2 \%$ were with scanty, $22 \%$ were with $1+, 52 \%$ were with $2+$, $24 \%$ were with $3+$ grades. Table 3 demonstrates the bleach method (ZN / LED) on negative smear grading from sputum samples. In $\mathrm{ZN}$ staining for bleach $12 \%$ samples showed positivity, while LED for bleach $18 \%$ scanty and $8 \%, 1+$ grading was directly smear negative samples.

Table.1 Rate of detection of Mycobacterium tuberculosis by direct sputum smear and after Bleach sedimentation

\begin{tabular}{|c|c|c|c|}
\hline $\begin{array}{c}\text { Stain of direct sputum } \\
\text { sample }\end{array}$ & $\begin{array}{c}\text { Total no.of } \\
\text { sample studied }\end{array}$ & $\begin{array}{c}\text { No. Of smear +ve } \\
\text { after bleach }\end{array}$ & P. Value \\
\hline $\begin{array}{c}\text { Smear+ve } \\
\text { Sample }\end{array}$ & 50 & $50(100 \%)$ & \\
$\begin{array}{c}\text { Smear-ve } \\
\text { Sample }\end{array}$ & 50 & $06(12 \%)$ & $<0.0001 *$ \\
\hline $\begin{array}{c}\text { Total } \\
\text { *z-test to compare two proportion used }\end{array}$ & $\mathbf{5 6}(\mathbf{5 6 \%})$ & \\
\hline
\end{tabular}

Table.2 Effect of Bleach sedimentation on smear for detection of Mycobacterium tuberculosis by $\mathrm{ZN}$ staining and LED

\begin{tabular}{|c|c|c|c|}
\hline $\begin{array}{c}\text { Status of direct } \\
\text { sputum sample }\end{array}$ & $\begin{array}{c}\text { Total No.of Sample } \\
\text { Studied }\end{array}$ & \multicolumn{2}{|c|}{ No. of smear +ve after bleach } \\
\cline { 2 - 4 } & 50 & $\mathbf{Z N}$ & LED \\
\hline $\begin{array}{c}\text { Smear +ve } \\
\text { Sample }\end{array}$ & 50 & $\mathbf{0 6}(\mathbf{1 2 \%})$ & $\mathbf{1 3}(\mathbf{2 6 \%})$ \\
\hline $\begin{array}{c}\text { Smear }-\mathrm{ve} \\
\text { Sample }\end{array}$ & $\mathbf{1 0 0}$ & $\mathbf{5 6}(\mathbf{5 6 \%})$ & $\mathbf{6 3}(\mathbf{6 3 \%})$ \\
\hline Total & & & \\
\hline
\end{tabular}


Table.3 Effect of Bleach sedimentation method on grading in smear positive sputum sample by $\mathrm{ZN}$ and LED

\begin{tabular}{|c|c|c|c|c|}
\hline \multirow{2}{*}{ GRADE } & \multicolumn{2}{|c|}{ ZN } & \multicolumn{2}{|c|}{ LED } \\
\cline { 2 - 5 } & Direct & $\begin{array}{c}\text { After } \\
\text { Bleach }\end{array}$ & Direct & $\begin{array}{c}\text { After } \\
\text { Bleach }\end{array}$ \\
\hline SCANTY & $06(12 \%)$ & $05(10 \%)$ & $05(10 \%)$ & $01(2 \%)$ \\
\hline $1+$ & $18(36 \%)$ & $13(26 \%)$ & $17(34 \%)$ & $11(22 \%)$ \\
\hline $2+$ & $19(38 \%)$ & $22(44 \%)$ & $21(42 \%)$ & $26(52 \%)$ \\
\hline $3+$ & $07(14 \%)$ & $10(20 \%)$ & $07(14 \%)$ & $12(24 \%)$ \\
\hline TOTAL & $\mathbf{5 0}$ & $\mathbf{5 0}$ & $\mathbf{5 0}$ & $\mathbf{5 0}$ \\
\hline
\end{tabular}

Table.4 Comparison of grading in smear negative sputum sample by bleach sedimentation ZN and LED

\begin{tabular}{|c|c|c|}
\hline Smear grading & ZN & LFD \\
\hline Scanty & $\mathbf{0 6}(12 \%)$ & $\mathbf{0 9}(18 \%)$ \\
\hline $1+$ & 00 & $\mathbf{0 4}(08 \%)$ \\
\hline $2+$ & 00 & 00 \\
\hline $3+$ & 00 & 00 \\
\hline TOTAL & $\mathbf{5 0}$ & $\mathbf{5 0}$ \\
\hline
\end{tabular}

Tuberculosis (TB) is one of the leading cause of morbidity and mortality worldwide, affecting one third of world's population. Geographically, the incidence is much higher in south East Asia (India and China) together account for nearly $40 \%$ of the global TB cases. $^{13}$

The global TB control programmed used direct microscopy for rapid detection of acid fast bacilli directly from sputum ${ }^{14}$.It is the simplest procedure currently available to detect AFB in clinical sample by ZN-Staining. ${ }^{2}$ But TB microscopy is associated with low and variable sensitivity. Therefore, the Mycobacteriology laboratories require an inexpensive and efficient concentrated method to diagnose smear microscopy ${ }^{15}$

In the present study, as already stated that the positivity rate was $63 \%$ using LED for bleach whereas $\mathrm{ZN}$ for bleach could achieve $56 \%$ positivity (Table 2), which is in concordance with Saroj Hooja et al., who reported positivity increased by $6.67 \%$ for $\mathrm{ZN}$ and $11.11 \%$ for LED microscopy. The reason for this may be, in mycobacteria after treatment with bleach might be attributable to changes in surface properties of the mycobacteria (Charge and hydrophobicity), and for denaturing of sputum constituents leading to flocculation and subsequent increased sedimentation rate of mycobacteria.

Several studies suggested that as the direct and bleach method in LED were increased the number of bacilli than the comparison of direct and bleach method in $\mathrm{ZN}$ microscopy. As for the direct and bleach method in LED improve the positivity of smear grading in sputum specimen from the comparison by $\mathrm{ZN}$ for direct and bleach method. In this present study at $\mathrm{ZN}$ 
for direct and bleach method, the 50 samples were positive, in which $12 \%$ and $10 \%$ were with scanty, $36 \%$ and $26 \%$ were with $1+, 38 \%$ and $44 \%$ were with $2+$ and $14 \%$ and $20 \%$ were with 3+, while if LED for direct and bleach method were also 50 samples were positive, in which $8 \%$ and $2 \%$ were with scanty, $36 \%$ and $22 \%$ were with $1+, 42 \%$ and $52 \%$ were with $2+$ and $14 \%$ and $24 \%$ were with $3+$ grades (Table 3). The reason for this may be, Bleach processing has been reported to facilitate identification of bacilli by providing a clearer microscopy field through concentrating bacilli by sedimentation resulting that the improvement of the grading (scanty, $1+, 2+, 3+$ ) compared as the direct method in ZN and LED. These results are also concordance by Sharon L. Reed et al., (27\%).

In contrast, after bleach sedimentation in LED microscopy were more effect on the improvement of the grading (scanty, $1+, 2+$ ) of sputum smear microscopy compared than the bleach sedimentation in $\mathrm{ZN}$ microscopy. These observations are also concordance by $\mathrm{P}$. H. Vishnu et al., (21.6\%).

The presence of bleach in negative samples was more effective for the detecting of smear positivity. As the bleach method in LED microscopy were increased the grading as compared than the bleach $\mathrm{ZN}$ microscopy in sputum specimen. In the present study, $\mathrm{ZN}$ for bleach, $12 \%$ samples showed positivity on negative sputum specimen, while LED for bleach, 26\% samples showed positivity on negative sputum specimen (Table 4). The reason for this may be as tubercle in bacilli are attached in mucous of sputum sample, some samples were very thick, then added the bleach, they digest the thick sputum samples, the bacteria come out the thickest part of the sputum specimen and increase the sensitivity of negative sputum sample. These result are supported by Ongkhammy et al., (33.5\%). in which similar result were obtained in LED microscopy. Considering the fact that direct LED was valuable method for the detection of smear positivity in the comparison than the direct ZN staining specially in smear negative sputum specimen. Some of the smears negative of the sputum specimen were improve the positivity in direct LED. In the present study, direct ZN stain showed $0 \%$ positivity for smear negative sputum smear, while direct LED showed $8 \%$ showed positivity on the negative sputum specimen. These observations are concordance by Roma Goyal et al., (14.69\%). The reason for this may be as, sometimes AFB was present in less number of the sputum specimen, and they are not visualized in direct $\mathrm{ZN}$ staining. But in LED, AFB are stands out brightly against the background and more fields can examined in short periods of time.

World health organization emphasizes the quality assurance of smear microscopy in global TB control programs. Besides smear microscopy quality assurance, the importance of sputum specimen quality has been also suggested.

In conclusion, concisely for detection of Mycobacterium tuberculosis LED is better than the $\mathrm{ZN}$ in direct method. Bleach sedimentation increases the smear positivity as well as grading, from the smear positive sputum samples for $\mathrm{ZN}$ as well as LED.

Bleach sedimentation is better method to detect Mycobacterium tuberculosis especially from the smear negative sputum samples. Bleach sedimentation, it is cheap, easy to perform and early to visualized brightly against the dark backgrounds. Another advantage of bleach sedimentation was bactericidal and adding bleach to sputum may sterilize it, potentially protecting staff from tuberculosis infection during processing samples.

\section{References}

1. World health organization TB fact sheet no.104 April 2005.

2. Rao KS, Kumar HA, Rudresh BM, Srinivas T, Bhat KH, A Comparative study and evaluation of serum adenosine deaminase activity in the diagnosis of 
pulmonary tuberculosis: Biomedical Research 2010; (2): 189-194.

3. Core programed cluster communicable disease and disease surveillance tuberculosis. who (internet) updated by July 2012. http://www.whoindia.org/en/section3/sect ion123.html

4. Global tuberculosis report 2013. WHO [Internet]. Available from:http://apps. who.int/iris/bitstream/10665/91355/1/978 9241564656_eng.pdf

5. Boujtita N. Concentration of Mycobacteria in Clinical Samples using the Thermo Scientific General Purpose Centrifuge with $8 \times 50 \mathrm{~mL}$ Individually Sealed Rotor. [Internet] Available from: https://static.

thermoscientific.com/images/D01739

6. Verma D, Siddique ME, Mittal V, Sharan J, Kishore S. Evaluation of serological and molecular methods over conventional methods in diagnosis of pulmonary and extra pulmonary tuberculosis. Journal of Evolution of Medical and Dental Sciences. 2012; 1(4):473-81.

7. Bonnet M, Gagnidze L, Guerin PJ, Bonte L, Ramsay A, Githui W, Varaine F. Evaluation of combined LEDfluorescence microscopy and bleach sedimentation for diagnosis of tuberculosis at peripheral health service level. PLoS One. 2011;6(5):e20175

8. Khatib SI, Williamson MT, R Singh, JM Joshi. Diagnosis of pulmonary tuberculosis by smear microscopy and culture in a tertiary health care facility.Biology and Medicine,4 (1):3236, 2012

9. Available http//www.who.int/tb/advisorybodies/stag _tb-report 2009 pdf. Accessed on 2011 Jan 06

10. Mutha A, Tiwari S, Khubnani H, Mall S. Application of bleach method to improve sputum smear microscopy for the diagnosis of pulmonary tuberculosis. Indian J Pathol Microbiol. 2005 Oct;48(4):513-7

11. Khubnani $\mathrm{H}$, Munjal $\mathrm{K}$ Application of bleach method in diagnosis of extrapulmonary tuberculosis. Indian $\mathbf{J}$ PatholMicrobiol.2005 Oct; 48(4):546-50.

12. Manual of Standard operating procedures (SOPs) intermediate reference laboratory for tuberculosis, culture of Mycobacterium tuberculosis and drug susceptibility testing on solid medium. Revised National Tuberculosis Control Programme Central TB Division, (Ministry of Health and family welfare).New Delhi.2009; Version No.01.01:40-43.

13. Global tuberculosis report 2012 who [internet] Available from www.who.int/tb/publication/global_report /gtbr12-main.pdf

14. Global tuberculosis report 2009 who [internet] Available from www.who.int/tb/publication/global_report /gtbr09-main.pdf

15. Biotec laboratories. A rapid bacteriophage assay for detection of mycobacterium complex in clinical samples. Instruction manual, Biotec, IPSwich, Biotec Fast Plaque $\mathrm{TB}^{\mathrm{TM}} 2000$.

16. Yoon SH, Lee NK, Yim JJ. Impact of sputum gross appearance and volume on smear positivity of pulmonary tuberculosis: a prospective cohort study. BMC Infect Dis. 2012;12:172

\section{How to cite this article:}

Suneet K. Yadav, R. Sujatha, D.N. Singh and Deepak Sameer. 2018. Evaluation of Bleach Sedimentation Technique in Detection of Mycobacterium tuberculosis by LED Microscopy. Int.J.Curr.Microbiol.App.Sci. 7(07): 3460-3466. doi: https://doi.org/10.20546/ijcmas.2018.707.401 